\title{
Complete paraplegia with diffuse osteolytic skeletal metastases: an uncommon presentation of carcinoma of the prostate
}

\author{
Akshat Gupta $^{1} \cdot$ Nitesh Gahlot ${ }^{1}{ }^{1} \cdot$ Poonam Elhence ${ }^{2}$ \\ Received: 10 February 2020 / Revised: 22 March 2020 / Accepted: 22 March 2020 \\ (c) International Spinal Cord Society 2020
}

\begin{abstract}
Introduction Skeletal metastases of advanced prostatic cancer are usually osteoblastic in appearance. Osteolytic secondaries are an uncommon entity. In such scenarios, it is important to differentiate prostatic metastases from other pathologies which give an identical radiological appearance, such as multiple myeloma. Another important point to remember is that neurological complications secondary to prostatic metastases are quite uncommon.

Case presentation We describe a man with advanced prostatic carcinoma who presented to us with an uncommon combination of sudden onset complete paraplegia associated with diminished sensation in the lower half of the body and diffuse osteolytic lesions in the entire skeletal system. On detailed workup, it was found that his prostatic specific antigen (PSA) was grossly elevated. Markers for multiple myeloma were negative. Accordingly, computerized tomography of the abdomen was performed. The latter revealed an enlarged prostate gland infiltrating into the bladder base. Histopathological analysis from the prostate as well as the bone marrow showed an identical picture thereby confirming our diagnosis. The individual underwent palliative spinal canal decompression following which he was put on anti-androgenic treatment. At one year follow up, he was ambulatory and able to walk with use of crutches.

Discussion To the best of our knowledge, this is the first reported case of osteolytic prostatic metastases presenting as complete paraplegia. In this situation, in addition to evaluation for prostate CA, we also recommend screening for multiple myeloma as the clinico-radiological profile of the two diseases are similar but their management is very different.
\end{abstract}

\section{Introduction}

Carcinoma of the prostate is the second most common cancer in men [1-3]. Overall, it is the fifth most common cancer worldwide [3]. Its propensity to spread to the skeletal system can be explained by both the "seed and soil" hypothesis of Paget as well as the anatomical hypothesis that states that tumour dissemination is determined by the vascular/lymphatic drainage of the primary malignancy (in this case, the Batson plexus of veins) [2, 4]. The bones most frequently involved include the vertebrae (especially lumbar), ribs and pelvic bones [2, 5]. However, spread to other

Nitesh Gahlot

doc.nitesh@gmail.com

1 Department of Orthopaedics, All India Institute of Medical Sciences, Jodhpur, Rajasthan 342005, India

2 Department of Pathology, All India Institute of Medical Sciences, Jodhpur, Rajasthan 342005, India parts of the body, such as lungs and liver, have also been reported [6]. The radiological profile of bony metastases of carcinoma prostate is predominantly sclerotic [1, 5]. Uncommonly, these lesions give an osteolytic appearance $[1,5,7-14]$. In such cases, it is important to differentiate metastases secondary to prostate cancer from other causes of diffuse osteolytic bony lesions, such as multiple myeloma, lymphoma, tuberculosis and secondaries from other malignancies such as the lungs, thyroid and kidneys[7, 15].

\section{Case description}

The individual was a 69 -year-old retired man who presented to the emergency room with the chief complaint of suddenonset bilateral lower limb weakness for 2-3 days. He was apparently well 2 months ago when he started experiencing pain in his lower back which was dull, aching in character, progressive and was only partially relieved on analgesics. The pain was not relieved in the reclining position. There was no history of any trauma, radiating pain or 


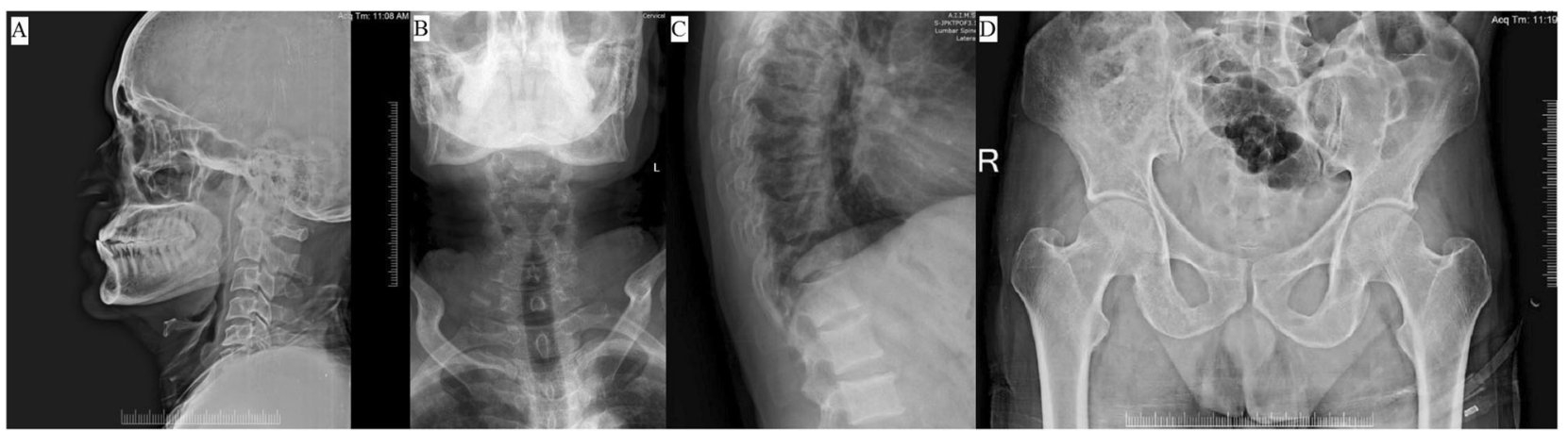

Fig. 1 Skeletal survey. $\mathrm{X}$ rays (a-d) showing diffuse, osteolytic lesions in the cervical and dorso-lumbar vertebrae, pelvic bones and left proximal femur.

Fig. 2 Contrast-enhanced computerised tomography (CECT) of the abdomen/pelvis (a-b). a An enlarged prostate gland [BLACK ARROW] infiltrating into bladder base with adjacently located \& thickened seminal vesicles [WHITE ARROWS]. b Enlarged para-aortic [BLACK ASTERISK] group of lymph nodes are also seen (color figure online).

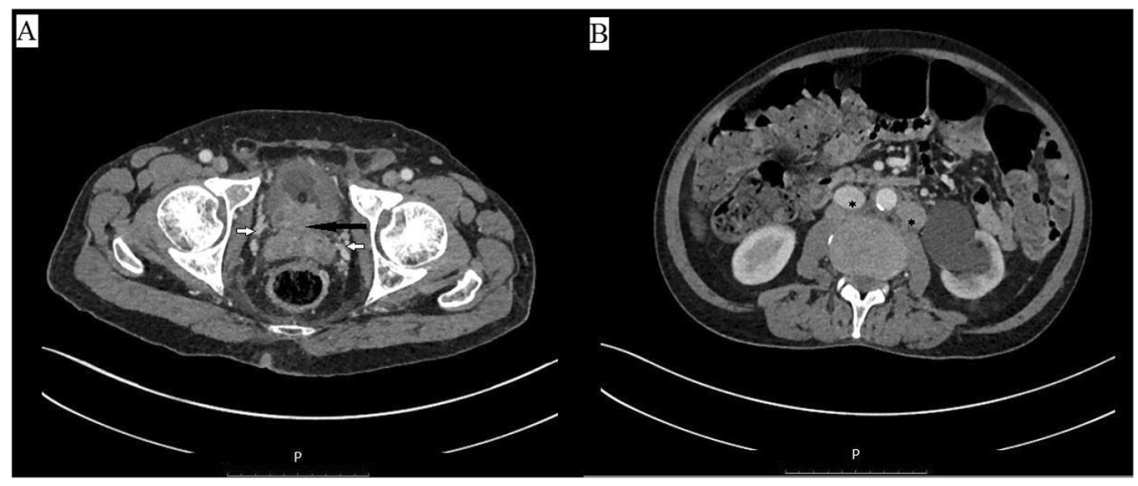

paraesthesias. Past medical history was unremarkable and there were no constitutional symptoms like anorexia, fever, etc. Clinical examination revealed diffuse tenderness over the spine, mainly in the thoraco-lumbar region. Neurological examination was carried out according to the International Standards for the Neurological Classification of Spinal Cord Injury (ISNCSCI) and revealed complete sensory as well as motor impairment L1 and below. Neurological level of injury was T12 AIS A. Higher mental functions were intact and bowel-bladder functions were within normal limits. Digital rectal examination revealed an enlarged, nodular prostate. All major lymph node areas were normal on palpation.

Radiological skeletal survey showed diffuse, osteolytic lesions in the vertebrae and pelvic bones (Fig. 1). Based on the individual's clinico-radiological parameters, the following differential diagnoses were considered: lymphoma, multiple myeloma, tuberculosis and metastases from a primary malignancy. The latter was evaluated by carrying out contrast-enhanced computerised tomography (CECT) scan of the chest, pelvis and abdomen (Fig. 2). It revealed an enlarged, hyper enhancing prostate with its median lobe extending into the bladder base. The surrounding seminal vesicles were thickened. Several deposits were noticed in the recto-vesical pouch with enlargement of the obturator, iliac and para-aortic groups of lymph nodes. Magnetic resonance imaging (MRI) of the spine showed presence of contrast-enhancing soft tissue masses replacing normal vertebral bodies at multiple levels, with an epiduralenhancing soft tissue component at T2-T3 as well as T7-T9. Compressive, myelopathic changes were seen in the dorsal spinal cord, with a pathological fracture at T11 (Fig. 3). A radionuclide bone scan with $\mathrm{T}_{\mathrm{c}}-99$ methyl diphosphate (MDP) showed increased tracer uptake in the sternum, bilateral ribs, bilateral scapulae, vertebral bodies, pelvic bones and the left proximal femur (Fig. 4).

Prostate specific antigen (PSA) was elevated at $>300 \mathrm{ng} /$ $\mathrm{ml}$, Erythrocyte Sedimentation Rate was $43 \mathrm{~mm} / \mathrm{hr}$, Creactive protein was $58.3 \mathrm{mg} / \mathrm{L}$ and serum alkaline phosphatase was $338 \mathrm{U} / \mathrm{L}$. Total and differential leucocyte count was within normal limits and no monoclonal bands were seen on serum protein electrophoresis. Urine for Bence-Jones proteins was also negative. Trans-rectal ultrasound (TRUS)-guided biopsy of the prostate revealed poorly differentiated adenocarcinoma cells arranged in sheets and a cribriform pattern (Gleason score $5+4$ ) with perineural invasion (Fig. 5). Bone marrow biopsy from the involved vertebrae showed an identical picture thereby confirming our diagnosis. There was no evidence of any caseating granuloma or abnormal blast cells in the histopathological report. Samples sent for Ziehl-Neelsen staining were negative. 
Fig. 3 Magnetic resonance imagine (MRI) of the spine (a-c). a Epidural enhancing soft tissue components at multiple levels with maximum cord compression at $\mathrm{T}_{2}$ level.

b Pathological fracture of $\mathrm{T}_{11}$. c Soft tissue masses [BLACK ARROWS] replacing normal vertebral bodies are also seen (color figure online).
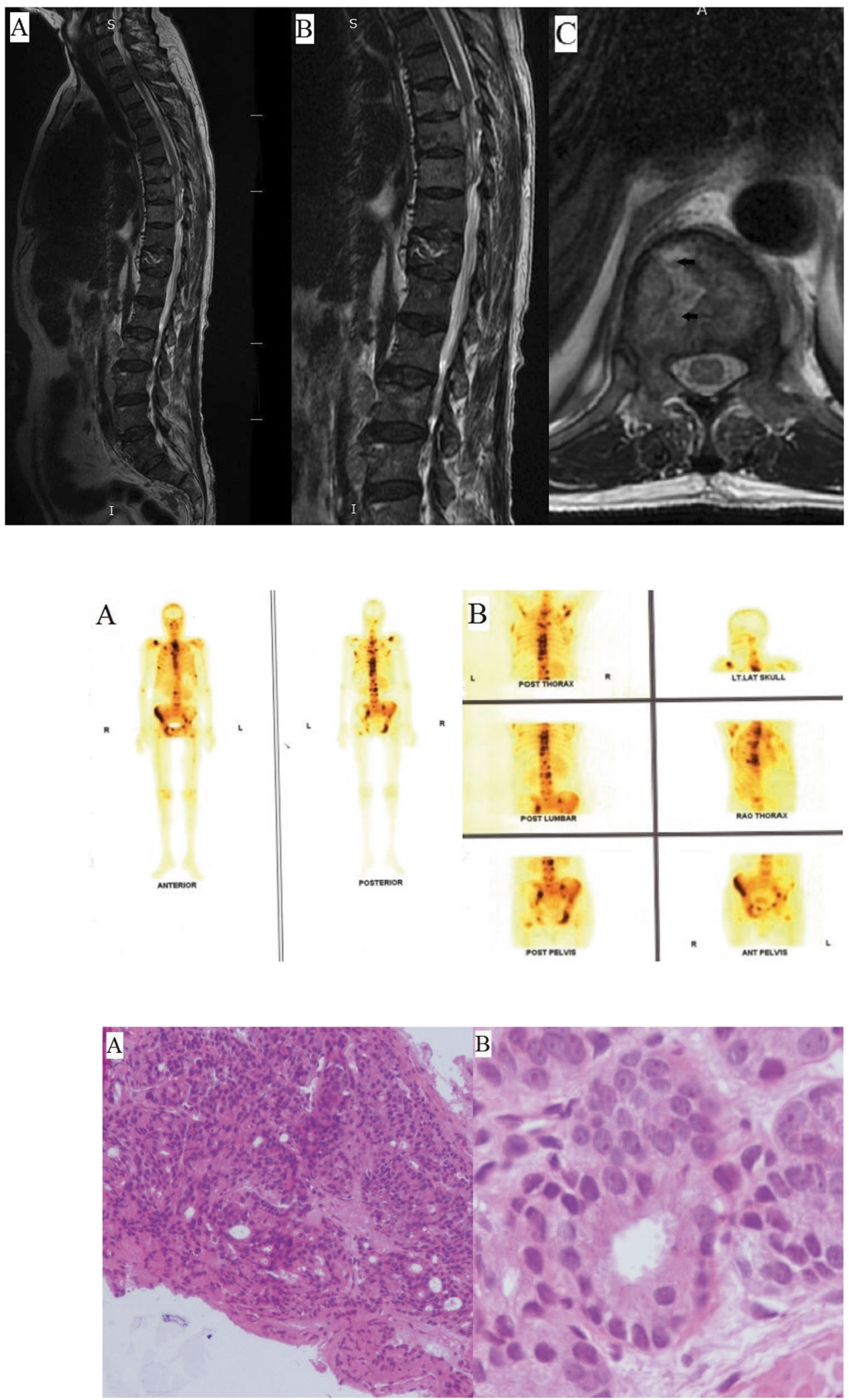

Fig. 4 Technetium-99 bone scan images (a-b). Increased tracer uptake seen in the sternum, bilateral ribs, bilateral scapulae, vertebral bodies, pelvic bones and left proximal femur.
Fig. 5 Histopathology slides (a-b). a Prostate core infiltrated by glands and strands of adenocarcinoma cells with hyperchromatic nuclei and moderate amounts of cytoplasm (H\&E/10x). b Tumour cell nuclei are enlarged, pleomorphic with prominent nucleoli (H\&E/ $40 x)$.
A diagnosis of carcinoma prostate with diffuse, osteolytic vertebral metastases (Tomita score 6) [16] was made. Hence, we performed palliative spinal canal decompression at the maximum level of compression (which was at the T2 vertebral level). Dorso-lumbar brace, analgesics and physical therapy were prescribed post-operatively. Anti-androgenic 
Table 1 A summary of all cases of carcinoma prostate presenting as lytic lesions in the skeletal system.

\begin{tabular}{|c|c|c|c|}
\hline Author (year) & Age/sex & Location & $\begin{array}{l}\text { Neurological deficit (for } \\
\text { spinal lesions) }\end{array}$ \\
\hline Migita et al. [11] (1999) & $62 / \mathrm{M}$ & Right proximal shaft humerus. & No information available \\
\hline Ansari et al. [13] (2003) & $60 / \mathrm{M}$ & Right proximal end of radius. & No information available \\
\hline Agheli et al. [12] (2009) & $70 / \mathrm{M}$ & Right intertrochanteric region and head of femur. & No information available \\
\hline $\begin{array}{l}\text { Rajendiran et al. } \\
\text { [5] (2011) }\end{array}$ & $65 / \mathrm{M}$ & $\begin{array}{l}\text { Diffuse osteolytic lesions involving multiple ribs, vertebrae with an } \\
\text { isolated, expansile lesion in the left iliac bone. }\end{array}$ & No deficit \\
\hline $\begin{array}{l}\text { Segamwenge et al. } \\
\text { [1] (2012) }\end{array}$ & $65 / \mathrm{M}$ & $\begin{array}{l}\text { Multiple osteolytic lesions involving the right proximal humerus, lumbar } \\
\text { spine, right proximal femur and pelvic bones. }\end{array}$ & No deficit \\
\hline Sharma et al. [9] (2013) & $67 / \mathrm{M}$ & Diffuse osteolytic lesions involving almost the entire skeleton. & No deficit \\
\hline Bakhsh et al. [14] (2015) & $80 / \mathrm{M}$ & $\begin{array}{l}\text { Multiple osteolytic lesions seen in the vertebral column as well as ribs with } \\
\text { a pathological fracture of } T_{5} \text { vertebrae. }\end{array}$ & No deficit \\
\hline Bird et al. [10] (2016) & $70 / \mathrm{M}$ & 11th rib & No information available \\
\hline Idowu [7] (2018) & $66 / \mathrm{M}$ & $\begin{array}{l}\text { Multiple lytic lesions seen in the skull, lumbosacral spine, pelvis and } \\
\text { bilateral proximal femur. }\end{array}$ & No deficit \\
\hline Alabed et al. [8] (2018) & $76 / \mathrm{M}$ & $\begin{array}{l}\text { Multiple lytic lesions involving the lumbosacral spine as well as the } \\
\text { pelvic bones. }\end{array}$ & No deficit \\
\hline Present study (2019) & 69/M & $\begin{array}{l}\text { Diffuse osteolytic lesions seen in the vertebral column, left proximal femur } \\
\text { and the pelvic bones with a pathological fracture of } T_{11} \text { vertebrae. }\end{array}$ & Complete paraplegia \\
\hline
\end{tabular}

therapy with gonadotrophin-releasing hormone (Gn-RH) agonists and palliative radiotherapy to the pelvis were performed. At 1-year follow up, the individual was ambulatory with crutches.

\section{Discussion}

Skeletal metastases have been reported in as many as 80-90\% cases of advanced carcinoma of the prostate $[1,2,7]$. The reason why the latter spreads to the skeletal system is supported by two theories. One is the "seed and soil hypothesis" of Paget, which states that prostatic tumour cells ("seed") adhere more readily to endothelial-cells derived from bone marrow ("soil") rather than those from other organs [4]. There is also an anatomical/mechanical basis in which the vertebral column is affected easily in metastatic prostate carcinoma due to dissemination of tumour cells via the Batson plexus of veins [1, 2].

Most skeletal metastases are osteoblastic on X-rays $[1,5]$. Mediators of osteoblastic activity include fibroblast growth factor, bone morphogenic protein, endothelin-1 as well as transforming growth factor- b [1]. However, there have been isolated cases reported in literature where secondaries from prostatic carcinoma have presented as osteolytic lesions [1, 5, 7-14]. In truth, prostatic metastases lead to activation of both osteoblasts and osteoclasts and the predominant type is affected by the specific interaction of the tumour with the bone environment [5]. Mechanisms described to account for osteolysis include overexpression of various cytokines and growth factors such as interleukins
1 and 6, parathyroid hormone related protein (PTHrp) as well as deregulation of the receptor activator of nuclear factor kappa- B ligand (RANKL) signalling pathway $[1,5,17]$. RANKL binds with its receptor and promotes osteoclastogenesis. Its activity is closely regulated by osteoprotegrin, which binds to RANKL and prevents it from binding to its receptor. Osteoprotegrin, therefore, prevents excessive resorption of the bones. Prostate cancer cells produce both RANKL and osteoprotegrin but the ratio between the two varies from case to case. When this ratio increases, it leads to excessive resorption of bones and appearance of diffuse osteolytic lesions [1, 5].

Metastatic carcinoma of the prostate with diffuse osteolytic lesions needs to be differentiated from similarappearing disorders, especially multiple myeloma [7, 15]. The two share several features. Both conditions have a similar clinical presentation and are commonly seen in African-American men in the same age group (usually around 60-70 years old). Treatment strategies are significantly different, thus accurate diagnosis is necessary. We relied upon several biochemical tests for this purpose. Complete blood counts showing pancytopenia, Bence-Jones proteins in the urine and a distinct $\mathrm{M}$ band in the globulin region on serum protein electrophoresis are all hallmark features of multiple myeloma [7]. In contrast, confirmatory diagnosis of other diagnoses such as lymphoma, tuberculosis, etc requires biopsy [7, 15].

In our patient, finding an enlarged, nodular prostate on digital rectal examination led us to suspect prostate carcinoma. Evaluation with CECT of the chest, abdomen and pelvis confirmed our suspicion. As all biochemical tests for 
multiple myeloma were negative, a biopsy was performed which revealed poorly differentiated adenocarcinoma in the prostate as well as in the bone marrow. There were no histopathological features to suggest tuberculosis or lymphoma.

The metastases were staged according to the classification given by Tomita et al. [16]. Based upon the histopathological grade of the primary malignancy, status of visceral metastases as well as extent of vertebral metastases, the lesions were given a score of six and palliative surgery was carried out.

Spinal canal compression is only seen in $7 \%$ of cases of metastatic prostate carcinoma and brain metastases are only seen in 1-2\% of cases [18]. To the best of our knowledge, this is the first reported case of carcinoma prostate with diffuse osteolytic vertebral metastases presenting with complete paraplegia secondary to spinal cord compression. A summary of all reported cases of lytic prostatic metastases and how they compare with ours is given in Table 1.

\section{Conclusion}

One must always have a high degree of suspicion when evaluating individuals with diffuse osteolytic skeletal lesions. Metastases from primaries such as prostate must always be ruled out, especially in elderly males.

Acknowledgements The authors wish to acknowledge and thank the patient for allowing them to publish his clinical data.

\section{Compliance with ethical standards}

Conflict of interest The authors declare that they have no conflict of interest.

Publisher's note Springer Nature remains neutral with regard to jurisdictional claims in published maps and institutional affiliations.

\section{References}

1. Segamwenge IL, Mgori NK, Yussuf SA, Mukulu CN, Nakangombe P, Ngalyuka PK, et al. Cancer of the prostate presenting with diffuse osteolytic metastatic bone lesions: a case report. J Med Case Rep. 2012;6:425.
2. Messiou C, Cook G, deSouza NM. Imaging metastatic bone disease from carcinoma of the prostate. Br J Cancer. 2009;101:1225-32.

3. Ferlay J, Shin HR, Bray F, Forman D, Mathers C, Parkin DM. GLOBOCAN 2008 v2.0, Cancer incidence and mortality worldwide: IARC CancerBase No.10. Int Agency Res Cancer. 2010. http://globocan.iarc.fr.

4. Langley RR, Fidler IJ. The seed and soil hypothesis revisited - the role of tumor-stroma interactions in metastasis to different organs. Int J Cancer. 2011;128:2527-35.

5. Rajendiran G, Green L, Chhabra G. A rare presentation of prostate cancer with diffuse osteolytic metastases and PSA of $7242 \mathrm{ng} / \mathrm{ml}$. Int J Case Rep Image. 2011;2:16-20.

6. Vinjamoori AH, Jagannathan JP, Shinagare AB, Taplin ME, Oh WK, Van den Abbeele AD, et al. Atypical metastases from prostate cancer: 10-year experience at a single institution. AJR Am J Roentgenol. 2012;199:367-72.

7. Idowu BM. Prostate carcinoma presenting with diffuse osteolytic metastases and supraclavicular lymphadenopathy mimicking multiple myeloma. Clin Case Rep. 2018;6:253-7.

8. Alabed YZ. Prostate cancer lytic bone metastases imaged with 18F-Fluorocholine PET/CT. Clin Nucl Med. 2018;43:220.

9. Sharma P, Karunanithi S, Dhull VS, Jain S, Bal C, Kumar R. Prostate cancer with lytic bone metastases: 18Ffluorodeoxyglucose positron emission tomography-computed tomography for diagnosis and monitoring response to medical castration therapy. Indian J Nucl Med. 2013;28:178-9.

10. Bird VY, Domino PM, Sutkowski R, Stillings SA, Trejo-Lopez JA. Prostate cancer with metastatic lytic bone lesions: positive bone scan post docetaxel chemotherapy in the setting of clinically successful treatment. Urol Case Rep. 2016;6:12-4.

11. Migita T, Maeda K, Ogata N. A case of prostate cancer associated with osteolytic bone metastases. Hinyokika Kiyo (Acta Urologica Japanica). 1999;45:371-4. in Japanese.

12. Agheli A, Patsiornik Y, Chen Y, Chaudhry MR, Gerber H, Wang JC. Prostate carcinoma, presenting with a solitary osteolytic bone lesion to the right hip. Radio Case Rep. 2009;4:288.

13. Ansari MS, Nabi G, Aron M. Solitary radial head metastasis with wrist drop: a rare presentation of metastatic prostate cancer. Urol Int. 2003;70:77-9.

14. Bakhsh MU, Lee S, Ahmad S, Takher J, Pareek A, Syed U, et al. Should prostate cancer be considered as a differential diagnosis in patients with osteolytic bone lesions? Eur Rev Med Pharm Sci. 2015;19:4791-4.

15. Nanda S, Bhatt SP, Steinberg D, Volk SA. Unusual cause of generalized osteolytic vertebral lesions: a case report. J Med Case Rep. 2007;1:33.

16. Tomita K, Kawahara N, Kobayashi T, Yoshida A, Murakami H, Akamaru T. Surgical strategy for spinal metastases. Spine 2001;26:298-306.

17. Rabbani SA, Gladu J, Harakidas P, Jamison B, Goltzman D. Over-production of parathyroid hormone-related peptide results in increased osteolytic skeletal metastasis by prostate cancer cells in vivo. Int J Cancer. 1999;80:257-64.

18. Benjamin R. Neurologic complications of prostate cancer. Am Fam Physician. 2002;65:1834-40. 